NBSIR 82-2486

\title{
The Measurement of Net Space Charge Density Using Air Filtration Methods
}

U.S. DEPARTMENT OF COMMERCE

National Bureau of Standards

Center for Electronics and Electrical Engineering

Electrosystems Division

Washington, DC 20234

April 1982

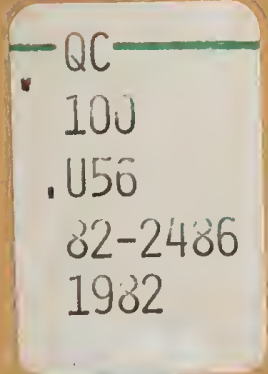

Prepared for:

Office of Electric Energy Systems

Department of Energy

Washington, DC 20585 

THE MEASUREMENT OF NET SPACE CHARGE DENSITY USING AIR FILTRATION METHODS

R. H. McKnight

U.S. DEPARTMENT OF COMMERCE

National Bureau of Standards

Center for Electronics and Electrical Engineering

Electrosystems Division

Washington, DC 20234

April 1982

Prepared for:

Office of Electric Energy Systems

Department of Energy

Washington, DC 20585

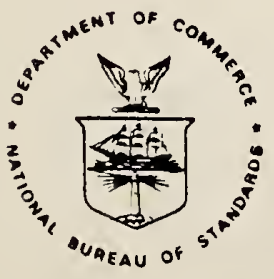

U.S. DEPARTMENT OF COMMERCE, Malcolm Baldrige, Secretary

NATIONAL BUREAU OF STANDARDS, Ernest Ambler, Director 

LIST OF FIGURES ....................... iv

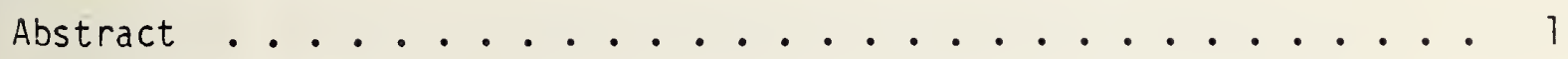

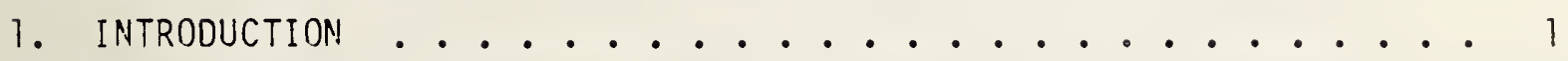

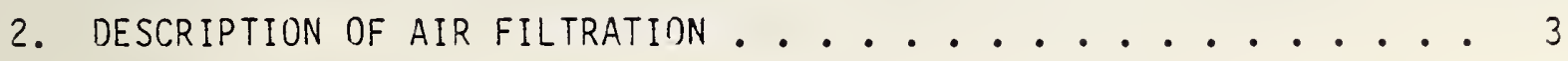

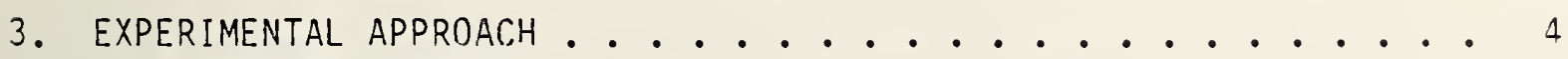

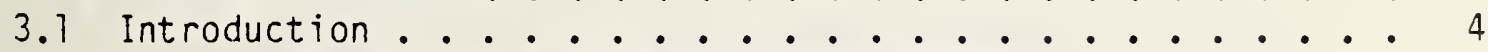

3.2 Measurements Using a Fiiter and Ion Counter ....... 8

3.3 Measurements Made Using Two Filters ......... 13

3.4 Accuracy of Measurements ............. 13

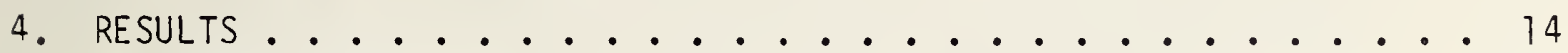

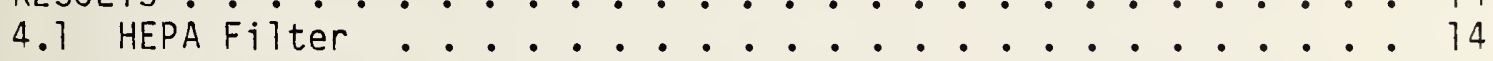

4.2 Dther Filters ........................ 17

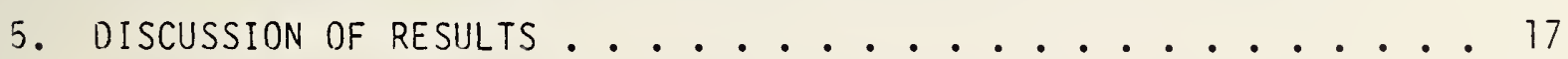

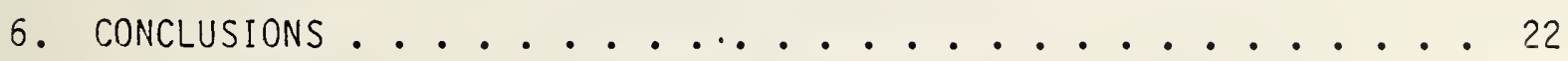

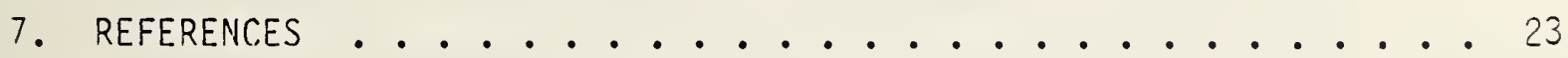


Figure 1. Experimental configuration for measurements in which the ion counter is used to measure transmitted charge density . . . . 5

Figure 2. Experimental configuration for measurements in which a filter is used to measure transmitted charge density . . . . . . . 6

Figure 3. Low speed air flow facility used to produce a test volume containing space charge. The filter and ion counter configuration is shown in place ............ . 9

Figure 4. Characteristic I-V curve for the ion counter obtained for a flow rate of $8.3 \times 10^{-3} \mathrm{~m}^{3} / \mathrm{s}$ and unfiltered inlet air. For this measurement, the positions of the ion counter and filter shown in figure 3 were interchanged. Also shown is the space charge detected by the filter following the ion counter . . . . . . . . . . . . . . . . . 11

Figure 5. Characteristic I-V curve for the ion counter obtained for a flow rate of $8.3 \times 10^{-3} \mathrm{~m}^{3} / \mathrm{s}$ and filtered inlet. air. The experimental arrangement was the same as for figure 4 . . . 12

Figure 5. Summary of HEPA filter transmission measurements. Shown for comparison are the result.s of Moore, et al., Paltridge, and the factory measured transmission (nOP test). Filter-filter measurements--nositive ions, o-negative ions. Filter-ion counter measurements--t-positive ions, x-negative ions. Filter-ion counter measurements (alpha source)-- -positive ions, 7-negative ions .............. 15

Figure 7. Transmission through steel wool filter. Flow facility inlet unfiltered-- -positive ions, o-negative ions. Filtered inlet-- -positive ions, $\Delta$-negative ions ....... 18

Figure 8. Transmission through commercial air filter. Flow facility inlet unfiltered-- -positive ions, o-negative ions. Filtered inlet-- 4 -positive ions, $\Delta$-negative ions ...... 19

Figure 9. Transmission through "furnace" filter. Flow facility inlet unfiltered-- 1 -positive ions, $\Delta$-negative ions. Filtered inlet--positive ions, o-negative ions....... 20 
THE MEASUREMENT OF NET SPACE CHARGE DENSITY

USING AIR FILTRATION METHODS

R. H. McKnight

The efficiency of a high efficiency particulate air (HEPA) or absolute filter for removing charge from an air stream has been measured for a variety of space charge and air flow conditions. Ion densities ranged from $10^{5}$ to $10^{6} / \mathrm{cm}^{3}$ and were for positive and negative space charge as well as mixtures. For all conditions studied, the transmission of the filter was less than $0.1 \%$. For space charge consisting predominantly of ions of one polarity, space charge density measurements made using HEPA filters and ion counters may be compared directly. The filter is well suited for all accurate measurements of net space charge density. Three other types of fibrous filters also have been studied.

Key words: corona discharge; HEPA filters; ion counters; ion density; ions; net space charge.

\section{INTROOUCTION}

The measurement of electrical quantities which depend on the ion density in air has been of interest to atmospheric electricity researchers for many decades. These measurements with others have provided information about various electrical processes in the atmosphere [1].1 More recently, similar measurements have been made to characterize the electrical environment in the vicinity of high voltage dc (HVDC) transmission lines [2]. In addition to providing information about external influences produced by the operation of such lines, these measurements offer guidance to those involved in experiments designed to investigate the biological effects of dc fields and ions.

For the effort described in this report, the electrical quantities of interest are the positive and negative space charge densities and the net space charge density. In the following discussion, the term space charge density will be used to refer to measurements of either the positive or negative charge per unit volume $\left(\mathrm{C} / \mathrm{m}^{3}\right)$ while net space charge density refers to the algebraic sum of the positive and negative space charge densities. It is possible to have near-zero net space charge density, but very large positive and negative space charge densities.

1 Numbers in brackets refer to the literature references listed at the end of this report. 
In the following discussion, the term "ion" will be used to describe molecular-sized ions which have mobilities of approximately $10^{-4} \mathrm{~m}^{2} / \mathrm{Vs}$. Other charge carriers, such as charged aerosols, have much smaller mobilities. The small ions are of concern in this study.

It is common in the literature to find discussion of ion density, that is, some number of ions per unit volume. This number is obtained from space charge density measurements by assuming each ion carries one electron charge. This is a reasonable assumption for molecular ions, but is not justified for charged particulates which are usually multiply charged.

Near the surface of the earth, the number of positive and negative ions varies considerably, but is typically less than $10^{9}$ ions per cubic meter. There is normally an imbalance between positive and negative ions, with a ratio of positive to negative ion density of about 1.35 . By contrast, near the ground under a HVDC transmission line or in a biological exposure system, the number density may be as large as $10^{12}-10^{13}$ ions per cubic meter. Because of this substantially larger ion density, the use of traditional measuring instruments and techniques under these more severe conditions may result in significant errors. The difficulty of the measurement problem is compounded by the presence of dc electric fields which may be as large as $50 \mathrm{kV} / \mathrm{m}$.

When using ion counters to measure space charge density, net space charge density may be obtained by taking the algebraic sum of the measured positive and negative space charge densities. If these quantities are nearly equal, there can be a large uncertainty in this difference. As a result, specialized techniques have been developed to measure net space charge density directly. The three most commonly used approaches involve air filtration methods, double field mills to measure the electric field gradient, and a determination of electrical potential caused by space charge by means of a Faraday cage. Each of these approaches is thoroughly described in the literature (the work of Vonnegut and Moore [3] presents an excellent summary of previous efforts).

Although 0bolensky [4] is usually first credited with using a filter technique to measure net space charge, Zeleny [5], in fact, used filtration methods to study the space charge distribution between two parallel plates as early as 1898. In the filtration method, a stream of air is drawn through a filter medium which is connected to ground through a sensitive ammeter. Some fraction of the charge in the air stream is removed by the filter. By measuring the volumetric air flow $\phi$ and the current I, the net space charge density is calculated as

$$
\rho_{\text {net }}=I / \phi .
$$


The net space charge density determined by (1) will be in error if a non-negligible fraction of the ions in the air stream passes through the filter.

Various filter materials have been used in previous investigations including cotton, steel wool, and different types of commercial filters. The highest efficiency filters available from commercial sources are high efficiency particulate air (HEPA) filters. These filters, sometimes called "absolute" filters, remove more than $98 \%$ of $0.3 \mu \mathrm{m}$ (micrometer) particulates from an air stream as measured in a standard test procedure. The filter material in these devices is a glass cloth made of very fine fibers. Yonnegut and Moore [3] first described the use of these filters in net space charge measurements. Other authors including Bent [6], Paltridge [7], and Moore, et. al. [8] describe their work with HEPA filters and each presents evidence that these filters exhibit a high efficiency for removing charge from an air stream. In each case, the conductivity of air was reduced by about $98 \%$ in passing through the filter. Filtration methods have also been used as space charge detectors in experiments designed to study charge generation during tank washing in large oil tankers [9] and in work concerned with downstream charge generation in air conditioning systems employing electrostatic air filters [10].

\section{DESCRIPTION OF AIR FILTRATION}

A vast literature exists which describes air filters and air filtration processes for many different operating conditions. There is much more experimental than theoretical work because of the complexity of the theoretical formalism describing particulate motion in a complex flow situation. There is little published research involving charged particles, electrified filters or both. For the purpose of the present work, a few general references will suffice and the interested reader may consult these works for further information. Discussions of filtration mechanisms and some earlier experimental results may be found in works by Chen [11], Green and Lane [12], and Dormon [13]. An early description of a high efficiency paper filter is given by Smith and Stafford [14]. Gillespie [15] has considered the role of electrical forces in the filtration process. When electrical forces are ignored, there are three distinct processes which are dealt with in discussions of filtration mechanisms. These processes are diffusion, interception, and inertia. The diffusion processes are most important for smaller particles. Because of the Brownian motion of the particles, they can diffuse across flow lines and impact the filter fiber. Larger particles are more likely to be filtered by an inertial process in which the particle is unable to follow the flow lines diverted by the filter fiber and is thereby captured. Interception results from the geometrical cross section of the filter fiber itself. Diffusion and inertial effects are functions of the speed of the particles, while all three depend on filter construction and particle size. 
If either or both the particles and the filter are charged, additional mechanisms arise because of various electrical forces as described by Gillespie [15]. In theoretical and experimental work reported in the literature, these forces increase the probability that a charged particle is intercepted by the filter.

It is not clear to what extent the available theory can be used to predict how effectively a filter will remove small ions from an air stream, since these are molecular-sized particles. In an exposure system, filtered air will probably be used with the result that there will be very few larger charged particles in the ion composition. Outdoors, near a HVDC line, it is quite likely that there will be substantial numbers of charged particulates. For these, existing filter data may be applicable.

\section{EXPERIMENTAL APPROACH}

\subsection{Introduction}

The purpose of this study was to determine the efficiency of various air filters for removing ions from an air stream passing through the filter for different air flows and space charge conditions. In principle, this measurement is made by determining the space charge density at the inlet and the outlet of the filter. Previous investigators [6-8] measured the change in conductivity of air before and after passing through the filter by using a Gerdien tube to measure conductivity. The present measurements were made using an experimental arrangement in which the filter under test was followed by a second device which was used to measure the space charge transmitted through the filter. The experimental arrangements used are shown in figures 1 and 2 , which are geometries using a filter and ion counter and two filters respectively.

The ion counter and the various filters used are expected to have responses which depend on the mobility spectra of the incident ions. This aspect of the experiment is discussed in more detail later, but by using two different techniques to detect the transmitted space charge it is possible to check for inconsistencies in the experimental procedure.

The transmission of the filter under test is defined as

$$
T(\text { percent })=\left(\rho_{T} / \rho_{i}\right) 100=\left(\rho_{T} /\left(\rho_{T}+\rho_{F}\right)\right) 100
$$

where $\rho_{T}$ is the transmitted charge density, $\rho_{i}$ is the incident charge density and $\rho$ is the charge density determined by the filter. 


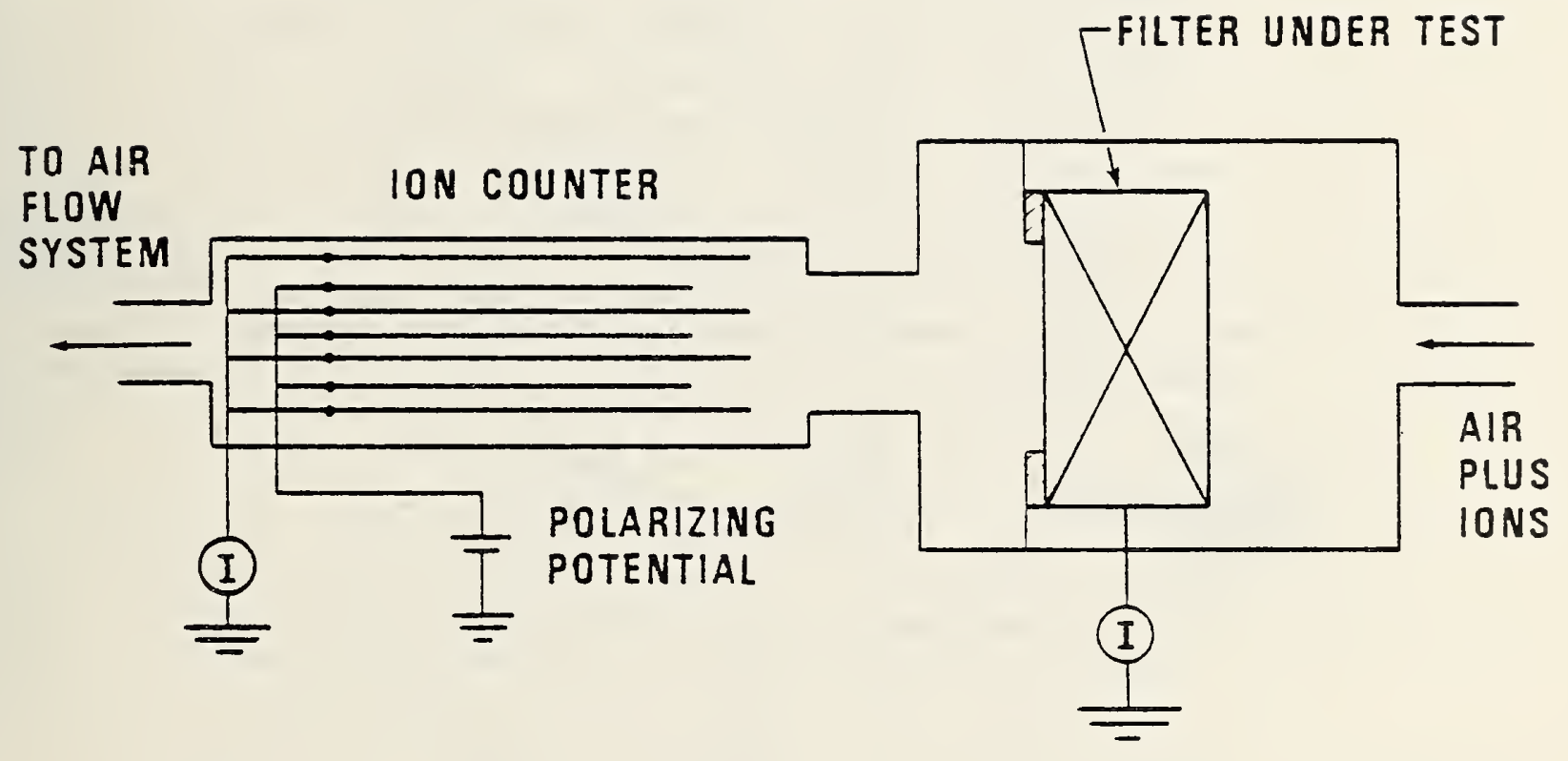

Figure 1. Experimental configuration for measirements in which the ion counter is used to measure transmitten charge density 


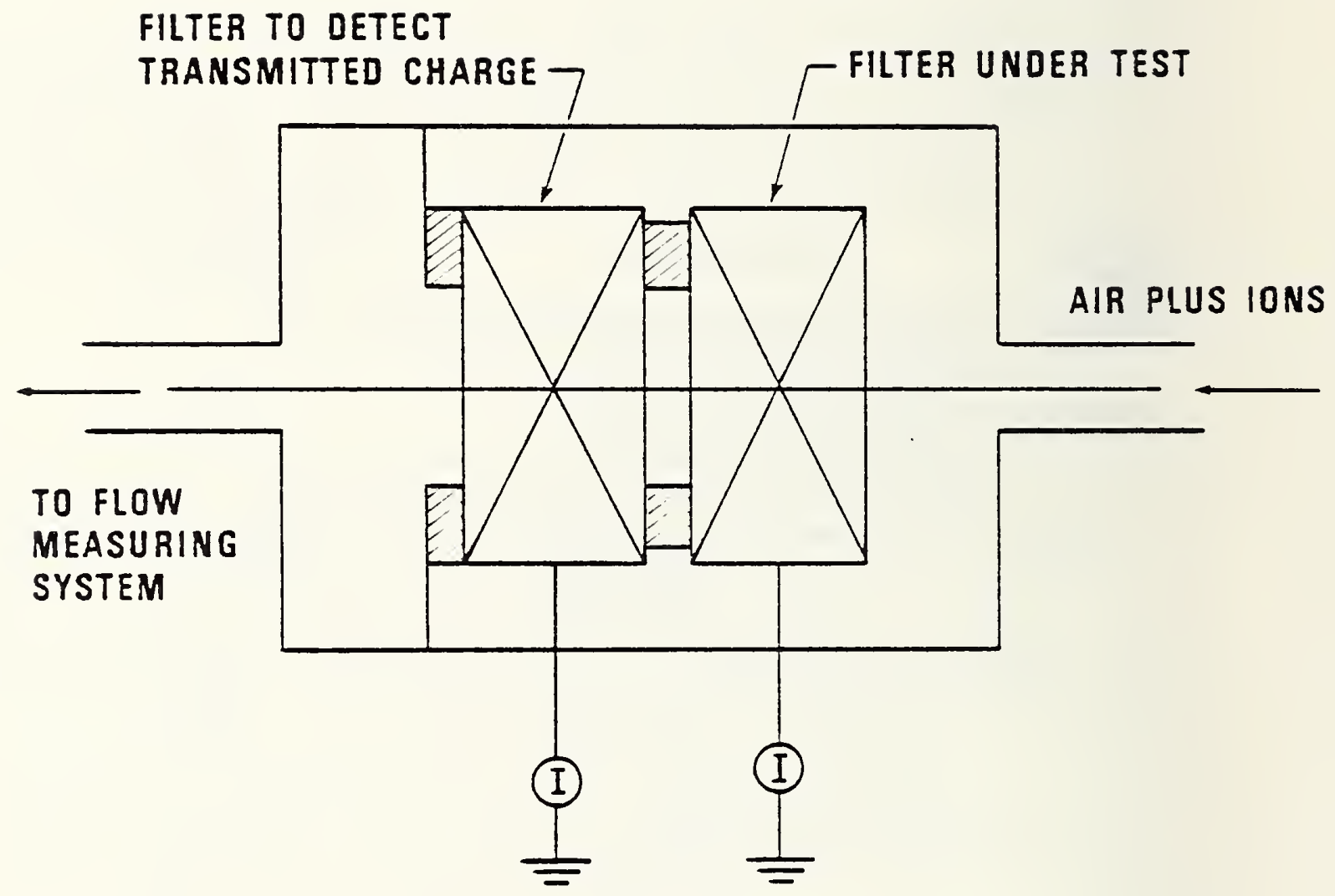

Figure 2. Experimental configuration for measurements in which a filter is used to measure transmitted charge density 
The air containing space charge is drawn from a test chamber in a low-speed air flow facility designed to produce a sizable volume containing a net space charge. This system, which is described elsewhere [16], is shown in figure 3. An air stream enters the inlet and passes through a series of screens to reduce turbulence. After leaving the screens, the air stream encounters a planar, coronadischarge, ion source. Nine vertical wires, $64 \mu \mathrm{m}$ in diameter, are located equidistant between two grounded screens. The wire-to-screen spacing is $0.1 \mathrm{~m}$. Ions are carried downstream from the ion source, through a transition and into the test section. It has been shown in earlier work that this facility can produce a space charge which is temporally smooth and spatially uniform to within a few percent over almost the entire cross sectional area of the test section. There is a gradual decrease in space charge density downstream from the source region due to ion losses to the walls. The ion source can be operated with either positive or negative dc supply and for the work presented here the operating voltage is $10 \mathrm{kV}$. Corona onset for both positive and negative voltages is between 5 and $6 \mathrm{kV}$. The space charge density produced in the test volume depends on the air speed at the ion source. Because the volumetric air flow through the system is much larger than that extracted by the absolute filter assembly, the perturbation on the $i$ on density produced by the sampling process is negligible. The inlet of the air flow facility has been configured so that a battery of HEPA filters can be inserted in the air stream. For this operating condition, the air passing through the corona region should be substantially free of particulates. In the present set of measurements, the use of the inlet filters resulted in a substantial reduction in air flow through the system. The fan used to move the air was unable to hand le the pressure drop across the filters (a new fan will be installed for future experiments). At the reduced air flow, the ion density was approximately $5 \times 104 / \mathrm{cm}^{3}$, and this led to experimental difficulties which are described later.

In a second set of measurements, the corona source was not used. Instead an alpha particle source was placed in the inlet tube leading to the filter assembly. By using this source, large positive and negative ion densities $\left(>106 / \mathrm{cm}^{3}\right)$ were obtained with near zero net space charge density. Therefore, the filter efficiency could be determined for a wide range of space charge density conditions.

The filter assembly consists of a commercial $20.3 \mathrm{~cm} \times 20.3 \mathrm{~cm}$ HEPA filter, mounted on an insulating support. All air flowing through the assembly passes through the filter. Current from the filter to ground is measured using an electrometer, and the volumetric flow rate was determined by means of a flowmeter. The air stream containing space charge enters the filter assembly through a $7.6 \mathrm{~cm}$ long tube $3.81 \mathrm{~cm}$ in diameter. Provision is made to mount a prefilter in front of the HEPA filter to reduce clogging. For measurements of the HEPA filter efficiency, the prefilter is removed. 


\subsection{Measurements Using a Filter and Ion Counter}

For the measurements made using the filter and ion counter (fig. 2), a parallel-plate ion counter [17] was configured to be in series with the filter assembly. Polarizing potential for the ion counter was provided by batteries and the current from the counter measured with an electrometer. The ion counter used was constructed in the laboratory and had been used in an extensive set of measurements so that its operation was well characterized. Counters of this type are extremely microphonic, and this defect, as discussed later, proved to be a severe limitation in the measurements described here.

The efficiency of the HEPA filter for removing space charge from an air stream was determined for positive and negative ions as a function of volumetric flow rate through the filter. For these measurements, the space charge was drawn from the test section (fig. 3), and the ion counter was used to detect the transmitted space charge. The initial measurements indicated that substantially less than $1 \%$ of the space charge density passed through the filter, confirming the results of earlier investigators.

The ions which make up the space charge have a range of mobilities. Since the response of both filters and ion counters to a space charge is mobility dependent, this results in some uncertainty in the measurements. During the course of the work described here, no means of determining the mobility spectrum was available in the laboratory.

Ion counters are characterized by a critical mobility. This quantity is defined as

$$
K_{C}=\varepsilon \phi / C V
$$

where $\varepsilon$ is the permittivity of air, $\Phi$ the volumetric flow rate, $C$ the counter capacitance and $V$ the polarizing voltage. All ions with mobilities equal to or greater than $K_{C}$ will be collected in the counter. A smaller fraction of less mobile ions will be collected as well. Some information about the ion mobilities can be obtained by varying the polarizing voltage and measuring the output current from the counter. In principle, the mobility spectrum can be obtained by manipulating the I-V data, but this process involves two numerical derivatives and is very uncertain. As a result the ion counter measurements were used only to provide some information about the mobility limits of the ions involved.

Characteristic I-V curves were recorded using the ion counter sampling directly from the air flow facility test chamber. These measurements were made for different flow rates through the counter. 


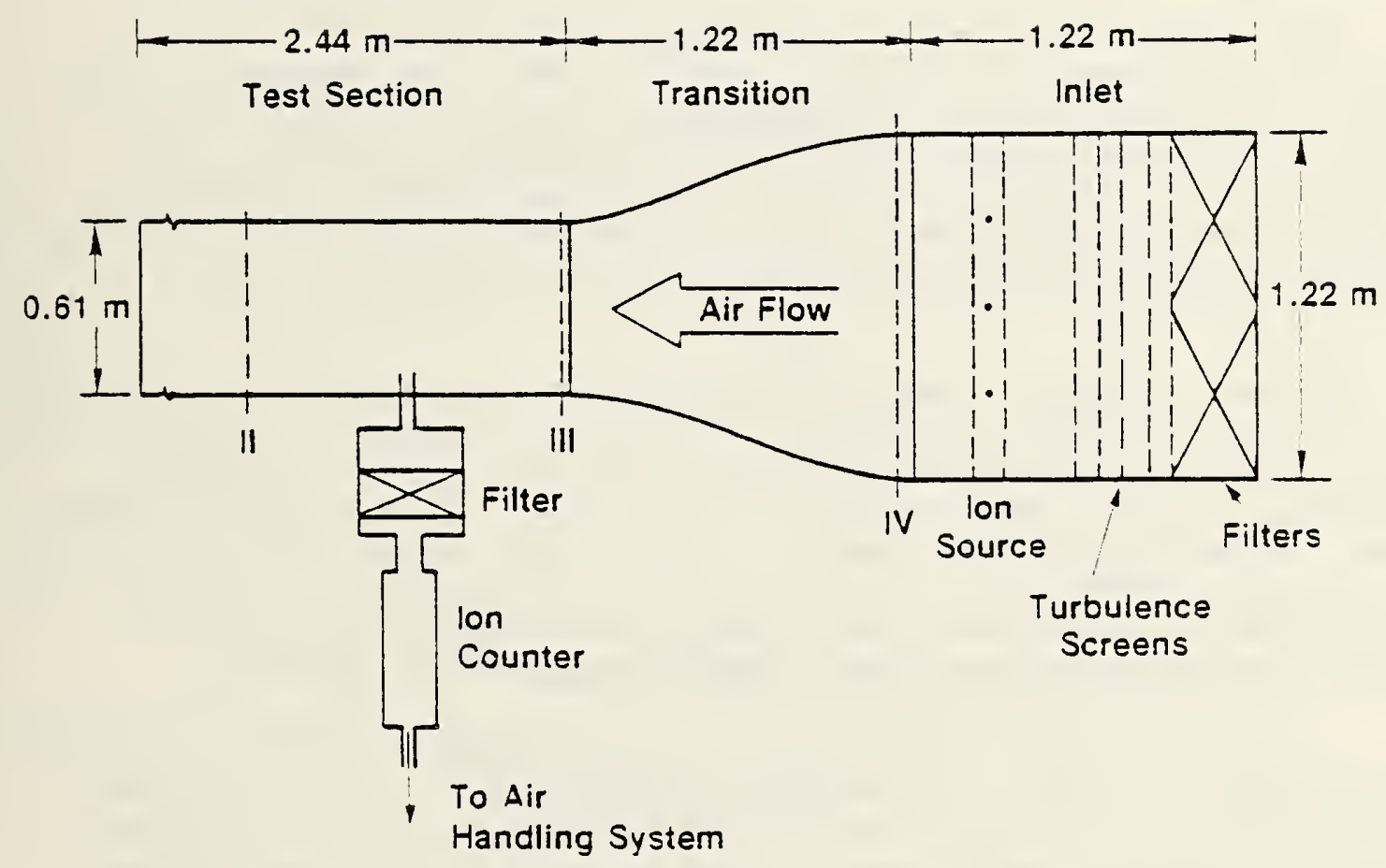

Figure 3. Low speed air flow facility used to produce a test volume containing soace charge. The filter and ion counter configuration is shown in place. 
Examples of the space charge density versus polarizing voltage curves obtained with the ion counter in front of the HEPA filter are shown in figures 4 and 5 . Here the difference is that the air entering the air flow facility was unfiltered for figure 4 , while in figure 5 the inlet air was filtered through HEPA filters. The charge density produced with filtered inlet air is substantially less than with no filters as was discussed earlier. The uncertainty in these measurements is less than $\pm 8 \%$.

The response of the ion counter has saturated at $20 \mathrm{~V}$ (volts) polarizing potential, but there is still a small ion component being detected by the filter. For the filtered inlet, this component is $2 \%$ and for the unfiltered inlet $8 \%$ of the total ion density. The substantial space charge indicated at the lowest polarizing voltage results from the field due to the space charge itself which causes ions to be precipitated to both the collecting plates and the polarizing plates.

Shown on figures 4 and 5 are the critical mobilities for different polarizing potentials. The small fraction of ions passing through the counter appear to have mobilities less than $0.005 \times 10-4 \mathrm{~m} 2 / \mathrm{Vs}$. A similar series of measurements was made for all the flow conditions used for the present experiments. In all cases, a polarizing voltage of $400 \mathrm{~V}$ was sufficient to ensure complete collection of any small ions passing through the filter. The polarizing voltage of $438 \mathrm{~V}$ used during the efficiency measurements was battery supplied.

Volumetric flow rates through the system under test were determined using either a turbine flowmeter or a hot wire mass flow transducer. Air speeds at the filter inlet were measured with a hot film anemometer system. During the measurements reported here, which extended over several months, the laboratory temperature varied from $21-23{ }^{\circ} \mathrm{C}$, and relative humidity, which was not controlled, ranged from 30 to $75 \%$.

For the measurements described above, the space charge was almost entirely of one sign. As indicated earlier, an alternative approach was taken to determine the response of the filter to conditions where there were large numbers of both positive and negative ions present, but in nearly equal quantities so the net space charge density was near zero. This condition was achieved by mounting a sinall alpha particle source in the inlet tube to the filter. The space charge density produced by the alpha source was determined by mounting the source at the ion counter inlet and maintaining the same air speed across the source as would be used in the transmission measurement. The space charge density determined by this measurement was used in eq (1) to determine the transmission of the filter for these conditions. Unlike the case of space charge consisting of ions of only one sign, the filter itself cannot be used to determine this information since it responds to the net space charge which for the alpha source is approximately zero. It was observed that the 


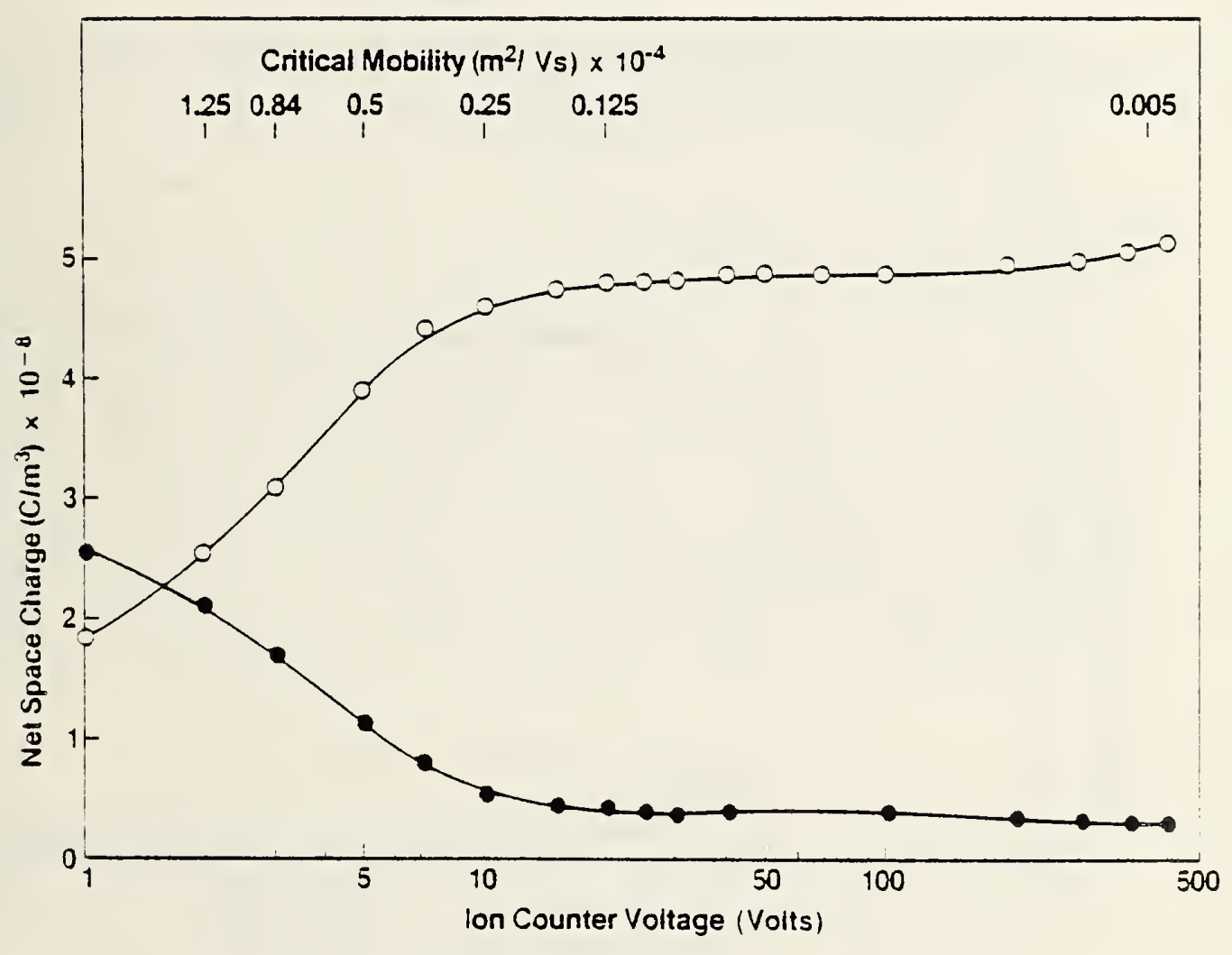

Figure 4. Characteristic I-V curve for the ion countar obtained for a flow rate of $2.3 \times 10^{-3} \mathrm{~m}^{3} / \mathrm{s}$ and unfiltered inlet air. For this measurement, the positions of the ion counter and filter shown in iigure 3 were interchanged. 1 lso shown is the space charge detected by the ilitar following the ion counter 


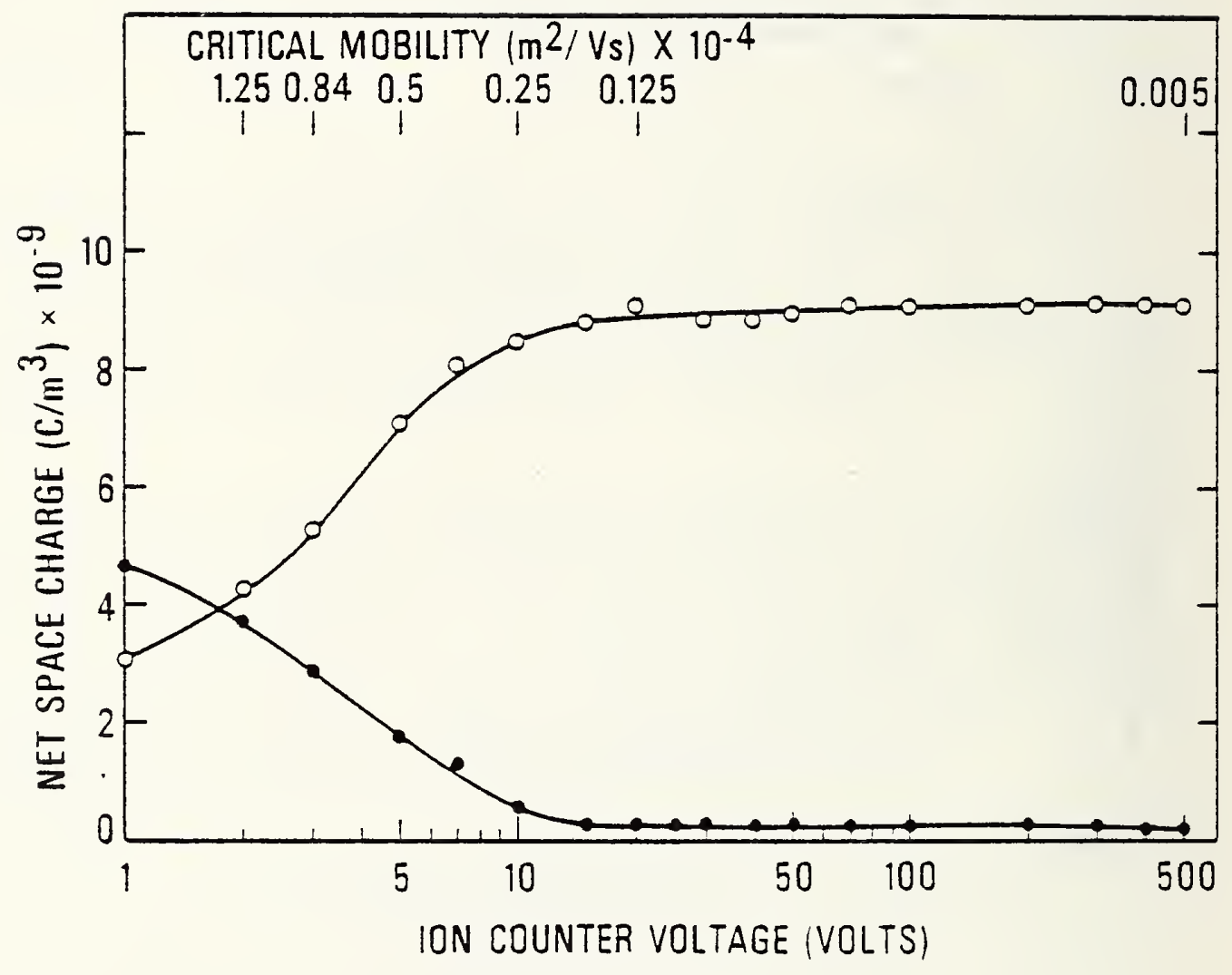

Figure 5. Characteristic I-y curve for the ion counter ohtained for a flow rate of $8.3 \times 10^{-3} \mathrm{~m}^{3} / \mathrm{s}$ and filtered inlet air. The experimental arrangement was the same as for figure 4. 
space charge density depended on the air speed near the source, but that for all conditions the positive and negative space charge densities were the same to within experimental uncertainty.

\subsection{Measurements Made Using Two Filters}

As noted above, the HEPA filter was found to have a transmission of less than $1 \%$ and was therefore suitable for use as a detector of transmitted space charge in a scheme designed to measure filter transmission as shown in figure 2. Transmissions of three different filters were measured using this method and these results are discussed later. It was found that one of the filters tested transmitted less than $10 \%$ of the incident ions for the test conditions of interest. As a result, the roles of the HEPA filter and the test filter were exchanged. The test filter then acted as the detecting filter in a measure of the HEPA filter transmission. This testing methodology, which appears at first to be circular, was used to check for possible inconsistencies in the experimental procedure. The ion counter and filter are expected to have mobility dependent response, and some evidence for this is seen in the results described below. The filterfilter method cannot be used where the incident space charge is not predominantly of one polarity, since a filter responds to the net space charge and is not polarity selective.

\subsection{Accuracy of Mea surements}

As noted earlier, the parallel plate ion counter is microphonic and as a result of vibration of the counter caused by both air flow through the system and mechanical coupling to the air flow facility, a noise signal was produced which was for some measurements a substantial fraction of the desired signal. Because of this, data were acquired by recording the electrometer output on a strip chart recorder and subtracting the background signal from the "ion-on" signal. For the lowest flow rates, where the current from the counter was smallest, this subtraction resulted in considerable uncertainty.

When the inlet was filtered, the available space charge density dropped by about a factor of 10. This reduction resulted in a transmitted space charge which was too small for meaningful measurements to be made when the HEPA filter was the device under test. As a consequence, there are no transmission results presented for a filtered inlet for the HEPA filter. For the other filters, which transmitted a larger fraction of the incident space charge, signal levels were high enough to obtain useful data.

Volumetric flow measurements have an estimated uncertainty of $5 \%$ and were made with a turbine flowmeter. Air speed measurements were made with a hot film anemometer operated near the lower end of the calibrated range. As a result, air speed values are subject to considerable uncertainty. Since the results displayed depend on volumetric flow rates and not directly on the air speed, there was no effort made to obtain an improved calibration. The electrometers used to measure currents are accurate 
to $3 \%$. For the filter-filter arrangement, there were no microphonic problems, but the detecting filter itself has a transmission which is uncertain to approximately $10 \%$. As a result of these various errors, the transmissions presented for the HEPA filter have relatively large uncertainty, which can be as large as $0.005 \%$ in the value for the transmission. This does not affect the usefulness of the HEPA filter, since the transmission of charge is so small that other sources of error dominate, i.e., measurement of volumetric air flow and current.

\section{RESULTS}

\subsection{HEPA Filter}

Three different types of measurements were made during the investigation reported here. They are (1) transmission through the HEPA filter using the ion counter as a transmitted space charge detector, (2) transmission through other filter materials using the HEPA filter as a detector, and (3) transmission through the HEPA filter using a second filter as a detector. The results from (1) and (3) will be presented together to facilitate discussion and maximize clarity. The initial set of measurements made using the filtercounter arrangement indicated that the HEPA filter transmitted substantially less than $1 \%$ of the incident ions for all conditions examined. Because of this, it was decided to use the HEPA filter in determining the transmission of three other filter materials. These transmissions were measured and one of the three filters (the commercial filter) was selected for use as a detector in a remeasurement of the HEPA filter transmission. During the course of the third set of measurements, the transmission of the filter was observed to increase substantially. This increase occurred after the filter system unit had been disassembled for maintenance. After this observation, the system was again opened and the filter carefully inspected for damage. There was no visual indication of change in the physical state of the filter. As a result of this, all earlier data were discarded and repeats were made of previous runs. Only the more recent data are presented here. It would be necessary to return the filter to the manufacturer for recertification to confirm that a small leak in the filter assembly had occurred. This information would not affect the present results but might offer an explanation for the change in transmission of charge.

All of the transmission numbers obtained using both filtercounter and filter-filter configurations are summarized on figure 6 . The data points displayed represent the average of several measurements and the width of the error bars are indicative of the spread in the data.

As noted earlier, the results presented here were determined in terms of volumetric flow rate and not directly as a function of the air speed at the face. The air speed values shown were measured using 


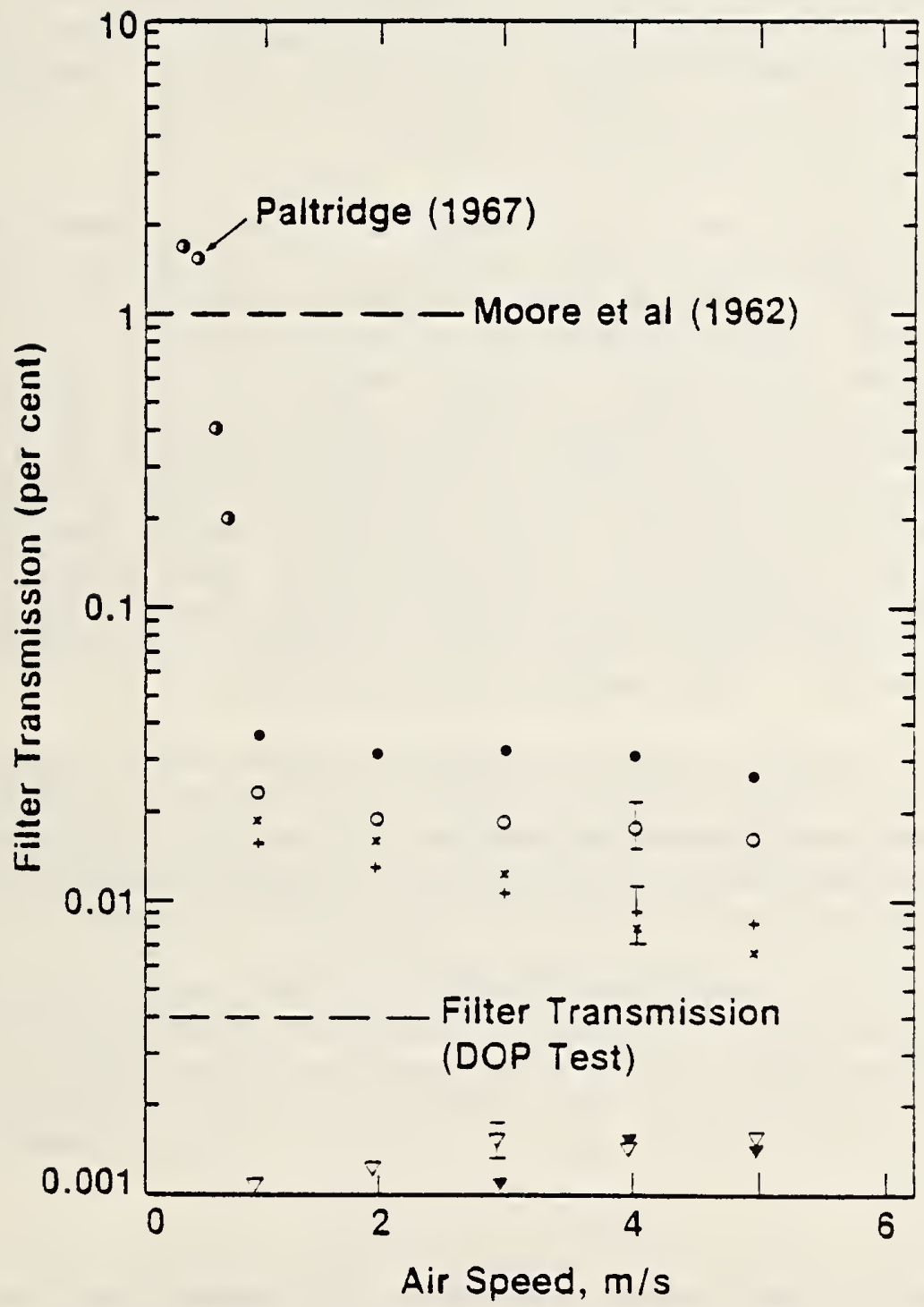

Figure 6. Summary of HEDA filter transmission measurements. Shown for comparison are the results of "oore et. a?, Daltridge and the factory measured transmission (DCP test). Filter-filter measurements---positive ions, D-negative ions. Fitter-ion counter measurements----positive ions, x-negative ions. Filter-ion counter measurement.s (alpha source)--P-positive ions, 7-negative ions 
a hot film anemometer near the lower end of the calihrated range. As indicated previously, these numbers are subject to considerable uncertainty. This uncertainty does not affect the results presented. The choice of face velocity rather than volumetric flow rate for use in presenting the data was made to facilitate comparison with previous data. Volumetric flow rates ranged from $(3.3-18.3) \times 10^{-3} \mathrm{~m}^{3} / \mathrm{s}$.

Transmission factors measured with the filter-filter arrangement were consistently higher than those obtained using the filter-counter configuration. There are a number of reasons for this observation. The second filter is tightly coupled to the HEPA filter so there are no ion losses between the filter elements. When the ion counter is used as a detector, there is a travel path of approximately $25 \mathrm{~cm}$ between filter and counter so ions can be lost by transport to the walls. The ion counter has a definite critical mobility for each operating condition so some lower mobility ions pass through the counter. The detecting filter may be more efficient at removing these ions from the air stream. As the volumetric flow rate decreases, the critical mobility becomes smaller, and there is some indication of convergence between ion counter and filter results as this occurs.

The lowest transmission factors were observed for the situation where the net space charge density was near zero, but the positive and negative space charge densities were high. A number of interesting features enter into explaining this result. Perhaps the most important is that for this space charge condition there is a very small contribution of slow ions to the total space charge. The air speed near the source is high ( 5 to $24 \mathrm{~m} / \mathrm{s}$ ) so little diffusional charging is expected since the particles spend little time in the space charge. There are no applied electric fields so there is no field charging. This situation is contrasted to that where the ions are produced in the corona source. Here both field and diffusion charging mechanisms are important [18].

If the surface of the filter becomes charged during exposure to a unipolar ion environment, electrostatic forces between the surface charge and an ion near the surface would tend to decrease the probability of collecting the ion. When the space charge is neutral, this mechanism would not come into play and an increased ion removal probability would be expected. There was indication of some charging of the filter during these measurements. During data acquisition, the current to the filter remained constant over periods of tens of minutes, which suggests that the charging was not large enough to cause a repulsion of ions at the front of the filter. However, when the ion source was turned off, the output current from the filter did not drop completely to zero. There was a small (less than 5\%) residual current which decayed to zero in a period of several minutes. No extensive investigation was made of this phenomena, since part of the current was presumably due to space charge in the laboratory recirculating through the air flow facility. 


\subsection{Other Filters}

Three other filter materials were examined to determine their usefulness in measuring space charge. These filters were (1) a densely packed steel wool filter $\sim 4 \mathrm{~cm}$ thick, (2) an inexpensive $2.5 \mathrm{~cm}$ "furnace" filter, and (3) a commercial filter of pleated design with a manufacturers' efficiency rating of $30-35 \%$ based on a standard test. For these measurements, the HEPA filter was used as a detector of transmitted charge. Figures 7,8 , and 9 show the transmission of these three filter materials determined for ion spectra produced with and without inlet filters on the air flow facility. The data plotted in figures 7 and 8 are the average transmission values determined for these filters for a 11 conditions studied. The error bars show a typical spread in the data, which were acquired over a several month period. In figure 9 , the data points represent the maximum transmission values observed, while the error bar illustrates the spread in data. There is a strong dependence on the nature of the ions heing measured (i.e., air flow facility inlet filtered or unfiltered), but except for the furnace filter little effect due to flow rate through the medium. These filters transmit a significant percentage of the incident ions, and would not be useful for accurate measurements.

The furnace filter shows a large variation with flow rate and also exhibited the greatest variability from run to run. This filter was operated substantially above its rated flow rates. The furnace filter was mechanically weak and very nonuniform. The results for this filter are present for comparison purposes only.

It is quite surprising that the transmission of ions through the steel wool and the commercial filter is as high as measured. The steel wool filter, in particular, was optically dense. However, the steel fibers making up the wool were not fine when compared to the fibers in other filters.

\section{DISCUSSION OF RESULTS}

The results presented here show clearly that for a wide range of space charge conditions and flow rates the HEPA filter may be used to accurately determine net space charge. For the particular filter studied here, the transmission of the filter for all conditions observed was less than $0.1 \%$.

A number of experimental difficulties reduced the overall accuracy of the measurements. As noted earlier, the parallel-plate ion counter used was microphonic. At the higher flow rates this resulted in a significant noise level with the large polarizing voltage used. The noise level combined with the low transmission of the filter to limit the quality of measurements obtained with the ion counter. As a result, it was not possible to determine an I-V curve for the ions transmitted through the filter. This measurement would have been extremely useful in estimating the mobility of the ions passing through the filter. 


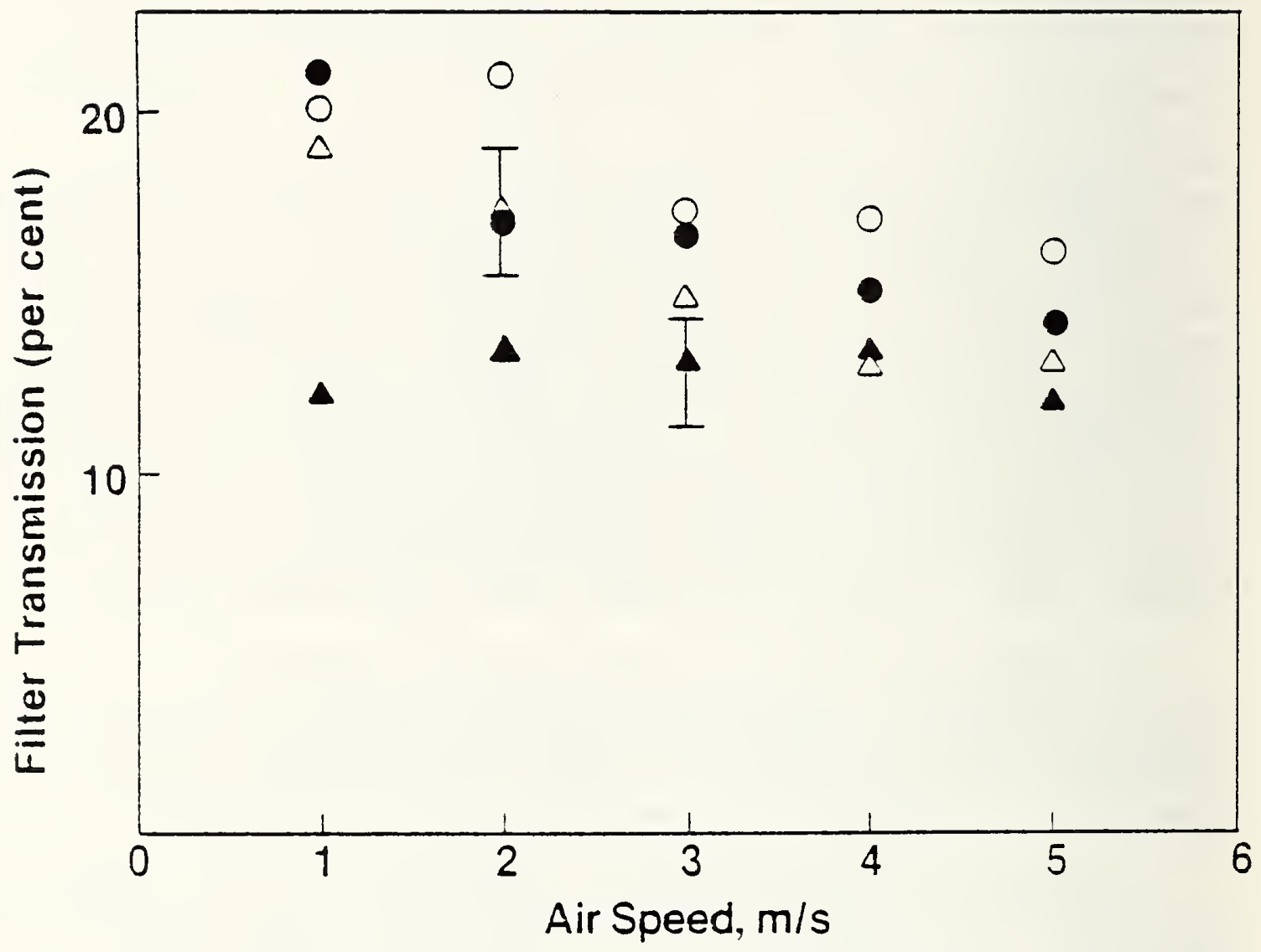

Figure 7. Transmission through steel wool filter. Flow facility iniet unfiltered-- o-positive ioris, o-negative ions. Filtered inlet--s-positive ions, $\Delta$-negative ions 


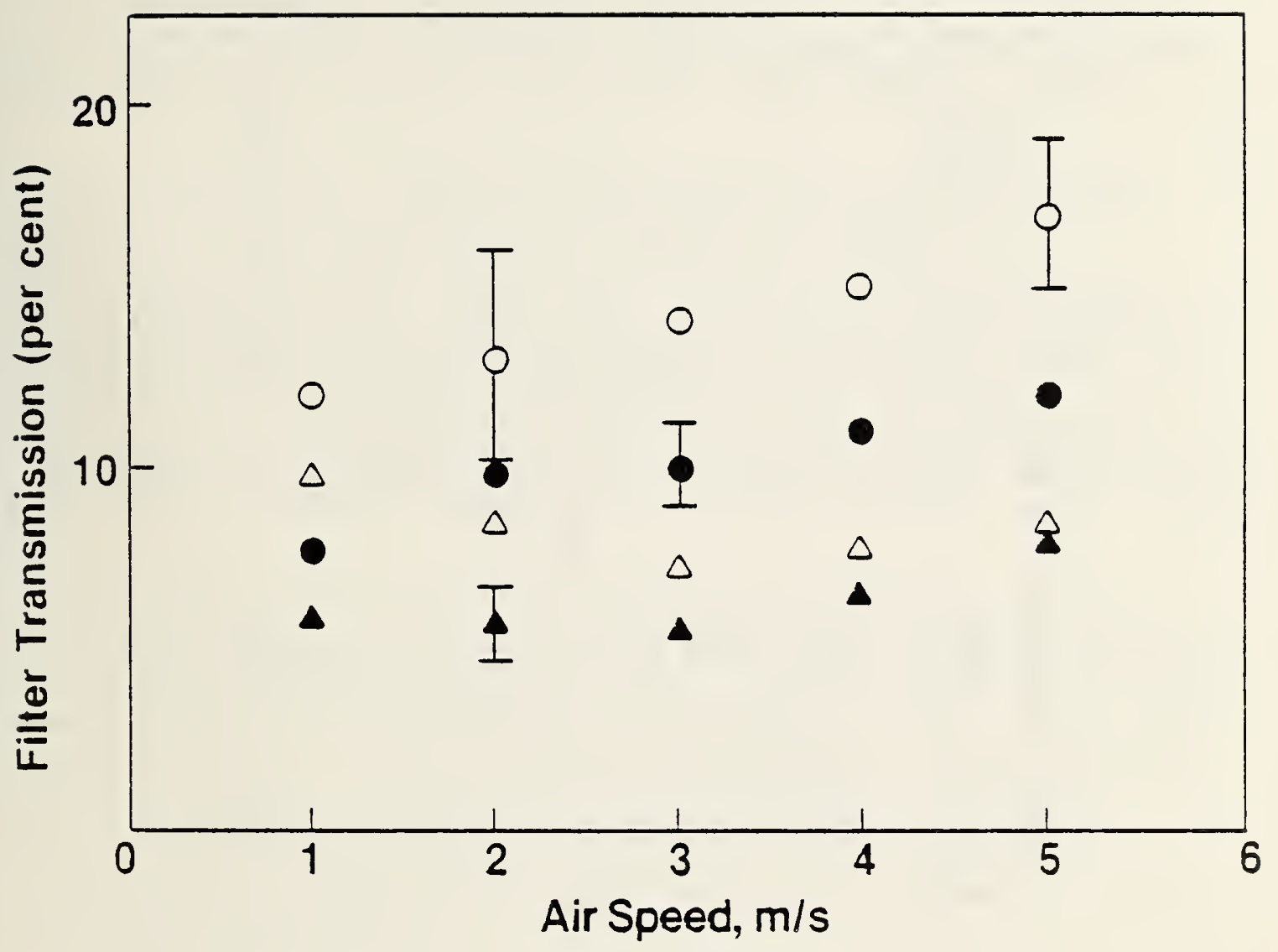

Figure 8. Transmission through commercial air filter. Flow facility inlet, unfiltered-- - - positive ions, o-nenative ions. Filuerer inlet--a-positive ions, 1 - negative ions 


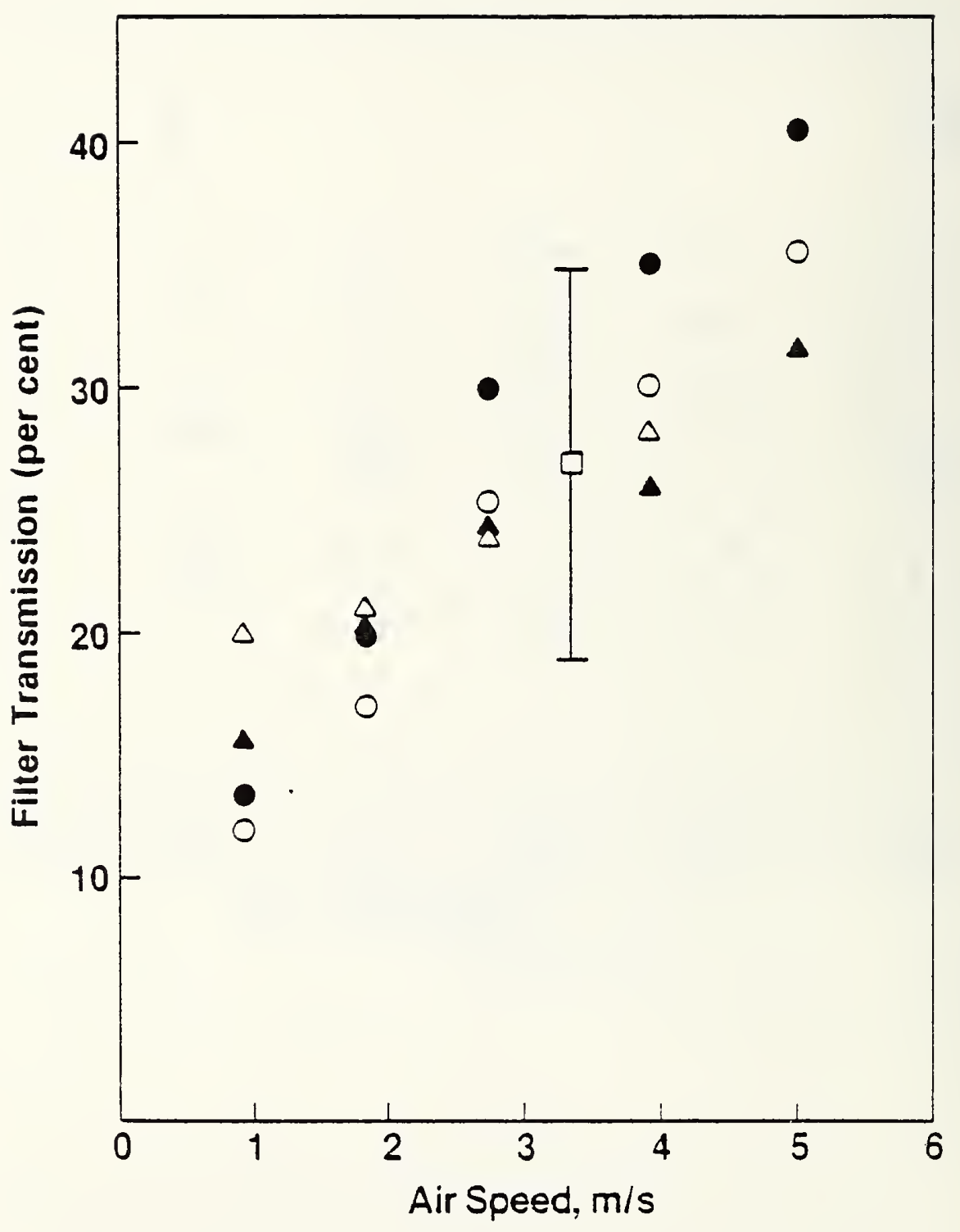

Figure 9. Transimission through "furnace" filter. Flow facility inlet unfiltered--L-positive ions, $\Delta$-neadive ions. Filtared inlet--o-positive ions, o-negative ions 
Considerable day-to-day variation was observed in the measured transmission. It is felt that this was a real effect and was a result of being unable to carefully control the composition of air entering the air flow facility. Although it was possible to place filters at the inlet of the facility to remove particulates, the resultant drop in net space charge produced in the test volume meant that reliable measurements could not be obtained for this operating condition.

The differences in observed transmissions as measured by the filter-ion counter and filter-filter technique appear to be due to differences in the response of the detector instrument. to the transmitted space charge. Resolution of this question would require an enhanced measurement capability which would result from substantial equipment modification and the development of a method for measuring the mobility spectrum of the ions in the test volume. From a practical standpoint, it is a moot point since net space charge measurements can clearly be made with less than one percent error being due to ion transmission through the filter for ions with the mobility spectra used here.

The mechanisms by which small ions are precipitated out by the filter are of interest. It is unlikely that inertia nlays a significant role for these molecular-sized ions. Diffusion would be expected to dominate with direct interception having only a small role. Roth processes would be affected by electrical forces. If electrification of the filter fiber is ignored, then the most likely electrical force involves the charge and its image force. If a particle of charge $q$ is located a distance $r$ from the center of a fiber of radius $R$ and dielectric constant $E$, the radial force on the particles due to an image charge is [14]

$$
F_{\Omega}=\frac{q^{2}}{16 \pi \varepsilon_{0}(r-R)^{2}} \frac{\left(\varepsilon_{0}-\varepsilon\right)}{\left(\varepsilon_{0}+\varepsilon\right)}
$$

where $\varepsilon_{0}$ is the dielectric constant of air. Paltridge [7] proposed that a distance $d_{0}$ be defined such that at this distance the average translational energy $K T / 2$ be equal to the potential energy (here, $K$ is Boltzmann's constant.)

$$
d_{0}=\left(r_{0}-R\right)=\frac{q^{2}}{4 \pi \varepsilon_{0} K T} \frac{\left(\varepsilon_{0}-\varepsilon\right)}{\left(\varepsilon_{0}-\varepsilon\right)}
$$

It is highly probable that an ion located $\left(r_{0}-R\right) \leqslant d_{0}$ from a fiber will move to the fiber and be neutralized. As a result, the effective radius of the $i$ on will be much greater than its physical radius. (It should be noted that similar considerations are encountered when dealing with charge transfer collisions between slowly moving ions and neutral atoms or molecules.) 
If the surface of the filter medium becomes charged (say on the surface) with the same polarity as the incident ion, then the situation becomes more complicated and the net force may in fact tend to inhibit the precipitation of the ion to the fiber. This may suggest a reason for the difference in transmission between a unipolar space charge and one in which the net space charge is effectively neutral. For the zero net space charge, it would be expected that the fibers would not on the average become charged. Because of this, there would be no repulsive force between ion and fiber which may be the case for a space charge made up of ions of one polarity.

One force that has not been described is that due to the space charge itself. At the high ion densities studied here, there is a significant electric field generated due to the space charge which will in effect cause the ions to separate and be forced to the filter material. Again for the zero net space charge condition this field is absent. In this case, however, the argument would say that the zero net space charge effect would be less than that for a unipolar charge density, which is opposite to what is observed.

It is clear that it is unlikely that a satisfactory description of the filter process will be forthcoming. The physical state of the surface of the filter material itself during the time that there is space charge near the filter is unknown. This lack of adequate theory, however, does not detract from the attractiveness of using the filter for net space charge measurements.

\section{CONCLUSIONS}

The efficiency of a HEPA filter for removing charge from an air stream has been measured for a variety of space charge and air flow conditions. For all conditions studied the transmission of the filter was less than $0.1 \%$. The mobility spectrum of the ions was not measured but the ions were predominantly small ions. There was some indication that there was a small amount of filter charging.

No adequate theoretical development is available to explain the results of these measurements. Diffusion is expected to be the dominant loss mechanism enhanced by electrical forces of which image forces should be the most important.

Other filter materials studied have transmissions of $5-15 \%$ and are not suitable candidates for accurate measurements.

Because of the low transmission of the HEPA filter, accurate measurements can be made of space charge densities where the ions are predominately of one sign. Under these conditions, measurements made using the filter and an ion counter may be directly comparable, depending on the mobility spectrum of the ions. Although much more work is needed, the HEPA filter appears to form the basis for a means of calibrating ion counters. 


\section{REFERENCES}

[1] Chalmers, J. A., Atmospheric Electricity, 2nd ed. (Pergaman Press, Oxford, 1967).

[2] Bracken, T. D., Capon, A. S., and Montgomery, D. V., Ground Level Electric Fields and Ion Currents on the Celilo-Sylmar \pm 400 KVDC Intertie During Fair Weather, IEEE Trans. PAS97, 1978, p. 370.

[3] Vonnegut, B. and Moore, C. B., A Study of Techniques for Measuring the Concentration of Space Charge in the Lower Atmosphere, (Final Report, Contract AF 191604), 1920, 31 Jan. 1958.

[4] Obolensky, W. N., Uber elektrische Ladungen in der Atmosphare, Ann. Phys. Lpz 77, 1925, p. 644 .

[5] Zeleny, J., On the Ratio of the Velocities of the Two Ions Produced in Gases by Rontgen Radiation; and on Some Related Phenomena, Phil. Mag. 46, 1898, p. 120.

[6] Bent, R. B., The Testing of Apparatus for Ground Fair Weather Space Charge Measurements, J. of Atm. and Terr. Phy. 26, 1964, p. 313.

[7] Paltridge, G. W., The Retention of Atmospheric Radioactivity by Fibrous Filters, J. of Geo. Res. 72, 1967, p. 1269.

[8] Moore, C. B., Vonnegut, B. and Mallabon, F. J., Airborne Filters for the Measurement of Atmospheric Space Charge, J. Geo. Res., 66 , 1961 , p. 3219.

[9] Owen, T. E. and Spiegel, R. J., Bulk Carrier Operations Safety Enhancement Project-Phase II, Volume II-Shiptank Electrostatic Model Studies, Final Report for Contract S-38044, U.S. Dept. of Commerce, 1978.

[10] Penney, G. W. and Hewett, G. W., Electrically Charged Dust in Rooms, Trans. AIEE 68,1949, p. 278.

[11] Chen, C. Y., Filtration of Aerosols by Fibrous Media, Chem. Rev. 55, 1955, p. 595.

[12] Green, H. L. and Lane, W. R., Particulate Clouds: Dusts, Smokes, and Mists, 2nd ed. E. \& F. N. Spon, Ltd. (Lond on), 1964.

[13] Dormon, R. G., Theory of Fibrous Filtration in High Efficiency Air Filtration, White, P. A. F. and Smith, S. E., editors, Butterworths (Lond on), 1964, p. 67. 
[14] Smith, W. J. and Stafford, E., Development of a Dry Fibrous Filter, in Air Pollution, MCCabe, L. C., editor, McGraw Hi11, 1952, p. 264.

[15] Gillespie, T., The Role of Electric Forces in the Filtration of Aerosols by Fiber Filters, J. Colloid, Sci. 10, 1955, p. 299.

[16] McKnight, R. H., Kotter, F. R., Misakian, M., and Ortiz, P., 1980 Annual Report: Electric and Magnetic Field Measurements, Nat. Bur. Stand. (U.S.), NBSIR $81-2267$ (1981).

[17] Knol1, M., Eichmerer, J. and Schon, R. W., Properties, Measurement and Bioclimatic Action of "Sma11" Multimolecular Atmospheric Ions, in Advances in Elec. and Electron. Phy., Marton, 1.. ed, 19, 1964, p. 178 .

[18] White, H. J., Industrial Electrostatic Precipitation, Addison-Wesley, 1963. 
SHEET (See in struction S)

4. TITLE AND SUBTITLE

The Measurement of Net Space Charge Density Using Air Filtration Methods

5. AUTHOR(S)

R. H. MCKnight

6. PERFORMING ORGANIZATION (If joint or other than NBS, see instructions)

NATIONAL BUREAU OF STANDARDS

DEPARTMENT OF COMMERCE

WASHINGTON, O.C. 20234

April 1982

9. SPONSORING ORGANIZATION NAME ANO COMPLETE AODRESS (Street, CI:. Stote, ZIP)

Department of Energy

Office of Electric Energy Systems

Washington, DC 20585

10. SUPPLEMENTARY NOTES

-Document describes a comouter program; SF-185. FIPS Software Summary, is attached.

11. ABSTRACT IA 200-word or less factual summary of most significanc informacion. If documenc includes a significanc oibliograohy or literature survey, mention ic here)

The efficiency of a high efficiency particulate air (HEPA) or absolute filter for removing charge from an airstream has been measured fof a varjety ${ }^{\text {of }}$ space charge and air flow conditions. Ion densities ranged from $10^{5}$ to $10^{6} / \mathrm{cm}^{3}$ and were for positive and negative space charge as well as mixtures. For a 11 conditions studied, the transmission of the filter was less than $0.1 \%$. For space charge consisting predominantly of ions of one polarity, space charge density measurements made using HEPA filters and ion counters may be compared directly. The filter is well suited for all accurate measurements of net space charge density. Three other types of fibrous filters also have been studied.

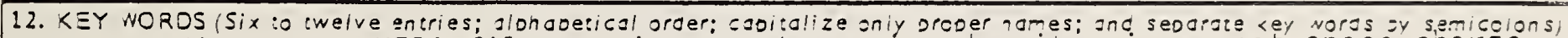
corona discharge; HEPA filters; ion counters; ion density; ions; net space charge.

- Order =rom Suderintencene of Documents, U.S. Government Pringing Office, Vasnington, Z.C. 20402.

14. NO.OF

DRINEDPAGES

28

Х Order Fiom National Tecinnical hiormation Service (NTIS), Soringieid, VA. 22'6 

$-\quad=$ 
